\title{
Automatized Approach to Assessment of Degree of Delamination around a Scribe
}

\author{
Petr Dolezel \\ Faculty of Electrical \\ Engineering and Informatics \\ University of Pardubice \\ Pardubice, Czech Republic \\ Email: petr.dolezel@upce.cz Email: pavel.rozsival@upce.cz
}

\author{
Veronika Rozsivalova \\ Metal Trade Comax \\ Velvary, Czech Republic \\ Email: \\ veronika.jaskova@mtcomax.cz
}

\author{
Jiri Tvrdik \\ Faculty of Electrical \\ Engineering and Informatics \\ University of Pardubice \\ Pardubice, Czech Republic \\ Email: jiri.tvrdik@student.upce.cz
}

\begin{abstract}
The aim of this paper is to select a suitable methodology and a sequence of procedures for assessment of degree of delamination around a scribe. This should be achieved with as high level of automation as possible. To keep the universality of application, regulation ISO 4628-8:2007 was followed. The procedure proposed in the paper implements an optical device for data acquisition and two approaches of image processing. It also provides a possibility of manual correction of the results to maintain the robustness of the solution. The preliminary evaluations of the results indicate, that more than $90 \%$ of the samples are interpreted correctly without any human operator interactions.
\end{abstract}

Keywords-Delamination; Optical System; Image Processing; ISO 4628

\section{INTRODUCTION}

All paint coatings degrade over time, no matter what they are exposed to. The resulting defects can be very diverse; for example, chalking, blistering, flaking or rusting of the painted metal. The evaluation of these defects must be defined in the most universal way possible, so that various involved entities, such as product vendors, can communicate efficiently with other interested parties. ISO 4628 "Paints and varnishes. Evaluation of degradation of coatings. Designation of the quantity and size of the defects and intensity of uniform changes to appearance" was proposed for the purpose of the assessment and quantification of the main defects that can occur in coatings [1].

ISO 4628 is a comprehensive regulation, which provides more than necessary number of tools for every possible type of coating defects. Nevertheless, manual evaluation of samples is implied [2], and, surprisingly, not many methods for automatized application of ISO 4628 have been presented, so far. Whitfield et. al presented an approach to quantify corrosion susceptibility based upon electrical resistance and colorimetric outputs [3]. A new concept of corrosion surface damage analysis by using the digital image processing is proposed in [4]. Other approaches, which use optical systems, are presented by Kapsalas et al. [5] or by Cringasu et. al [6]. The issue of cited works is the focus only for a limited range of materials or surfaces. Conversely, industrial entities ask for robust systems with a wide range of scope. Some techniques applied to different phenomena, which could be promising to use to the discussed issue, have been published, too [7], [8], [9].
Based on these facts, the aim of this work is to develop and present an approach for application of one part of ISO 4628 to a wide range of coatings, regardless of color, asperity or reflectivity.

In the following paragraphs, the aim of the our project is properly defined, the methodology of the assessment method is described, the solution is proposed and, eventually, several examples are presented.

\section{THE AIM OF THE PROJECT}

Producers of coating compositions need to test their products in various corrosive environments. One of the regulations, which defines the degree of coating degradation, is called "Assessment of degree of delamination and corrosion around a scribe" (ISO 4628-8:2007). This standard defines the criteria for evaluating both intensity and quantity of the delamination of paint coating or corrosion of the covered metal. A numerical scale of 0 to 5 is adopted for evaluation, where 0 signifies the absence of changes, while 5 means defects so notable that further discrimination is not reasonable [10].

The testing procedure starts with sample preparation. A set of metal plates is covered with coating composition and, after some time, a series of testings is performed; an assessment of degree of delamination around a scribe is one of them. Thus, the plates are scribed with a sharp edge and exposed to a corrosive environment for a defined period of time $(120 \mathrm{~h}$, $240 \mathrm{~h}, 480 \mathrm{~h}, 720 \mathrm{~h}, \ldots)$. After exposure, the plates are rinsed with tap water and the residues of water are then removed using compressed air. A loose coating is cut off with a knife blade held at an angle. A boundary should be situated where the coating becomes tightly adhered to the plate - see Figs. 1 to 3 for some examples of coated metals prepared by this procedure.

The area of delamination could be then determined by measurement and calculation or by comparison to pictorial standards. The issue is, that the degree of delamination is determined either manually, or by the autonomous system, which is, however, prepared only for one type of coating. Therefore, the project, which should provide a general autonomous tool for the degree of delamination assessment, has been set. The resulting device is presented in this paper. 


\section{Methodology}

According to ISO 4628-8:2012 [10], two possibilities of the assessment of degree of delamination around a scribe can be implemented.

\section{A. First option}

The width of the area of delamination has to be measured at a minimum of six points uniformly distributed along the scribe. With that, the arithmetic mean is determined and the resulting value is designated as the mean overall width of the zone of delamination, $d_{1}$, in millimeters.

Then, the degree of delamination $d$, in millimeters, can be calculated using the equation

$$
d=\frac{d_{1}-w}{2},
$$

where $w$ is the width of the original scribe, in millimeters. See Fig. 4 for an example of application.

\section{B. Second option}

The area of delamination should be determinable for this approach. The norm proposes laying transparent millimetergrid paper over the plate and counting the number of squares corresponding to the area. Then, the degree of delamination $d$, in millimeters, can be calculated using

$$
d=\frac{A_{d}-A_{l}}{2 l},
$$

where $A_{d}$ is the area of delamination, including the scribe area, in square millimeters, $A_{l}$ is the area of the scribe in the area evaluated, in square millimeters, and $l$ is the length of the scribe in the area evaluated, in millimeters. See Fig. 5 for an example of application.

\section{IMPLEMENTATION}

Apparently, the second approach of the degree of delamination assessment described in section III-B is more accurate and the procedure for acquiring the black and white image shown in Fig. 5 seems to be feasible. Hence, implementation of the second approach is tempting. However, after extensive literature research as well as after many experiments, we were not able to find a robust enough image processing technique,

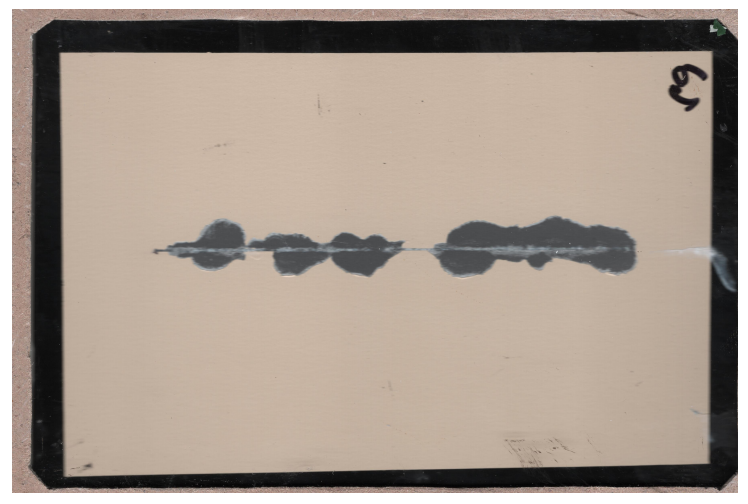

Fig. 1. Example 1.

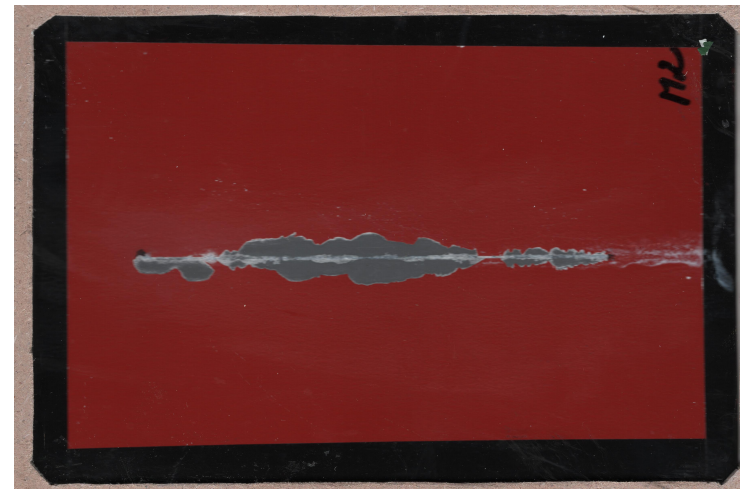

Fig. 2. Example 2.

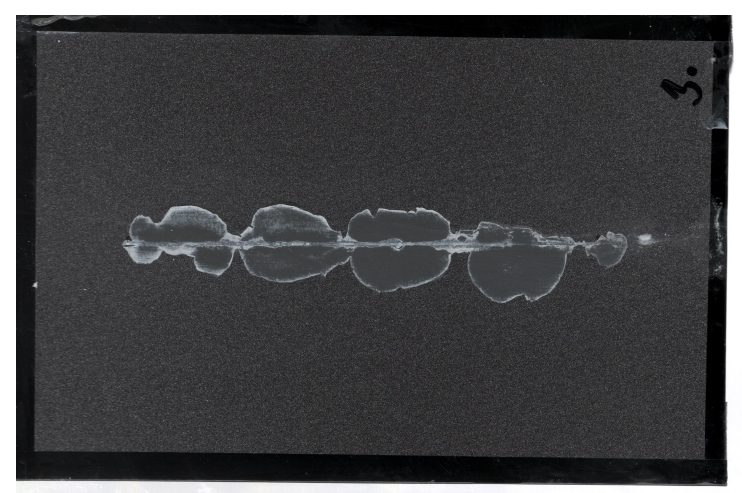

Fig. 3. Example 3.

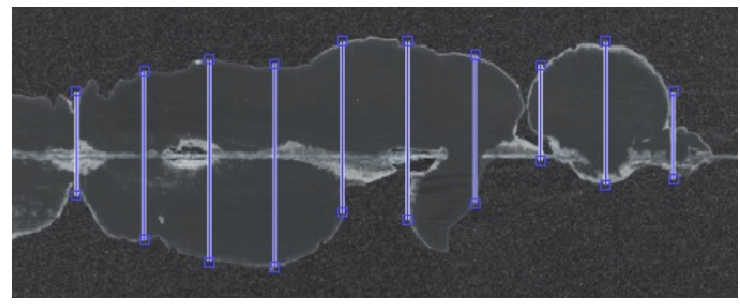

Fig. 4. Mean overall width of the zone of delamination is, in this case, defined as a sum of the lengths shown in the figure divided by ten.

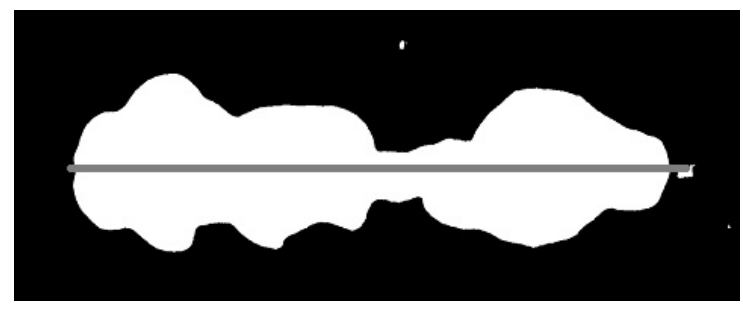

Fig. 5. White area is the area of delamination, gray is the area of the scribe.

which can provide a correct black and white image regardless of color, asperity or reflectivity of coating. Therefore, the intended device should provide a functionality for possible corrections performed by a human operator. And, a considering user friendly interface for these corrections, it would be much more convenient for the human operator to operate with a finite number of abscissae (Fig. 4) rather than with continuous areas (Fig. 5). 
In consequence, the first approach (section III-A) is selected for implementation. A block diagram of the intended device is shown in Fig. 6.

A generic color scanner is used for image acquisition in a first prototype of the device. After that, the acquired image has to be transformed into a black and white form, where white color represents the area of delamination. Clearly, this is the most important step of the whole procedure. After many experiments and using a trial and error method, two possibilities of this transformation were implemented into a control software.

The first approach implements an active contours method without edges proposed by Chan and Vese in 2001 [11]. This complex approach was initially designed for object detection, and it is based on techniques of curve evolution, MumfordShah functional for segmentation, and level sets. Adversely, the other approach is based more on "brutal force" and it implements a set of very basic image processing operations:

- Subtracting the image from the image acquired at the beginning of the process. The result should roughly represent the area of delamination.

- Transformation of the image into a grayscale image. To be specific, an intensity image is determined, where grayscale values I are computed by forming a weighted sum of the R, G, and B components

$$
\mathrm{I}=0.2989 \mathrm{R}+0.5870 \mathrm{G}+0.1140 \mathrm{~B} .
$$

- $\quad$ Filtration of the image using 2D median filtering [12], where each output pixel contains the median value of neighboring pixels in the input image. The size of neighborhood corresponds with the size of the original image; $0.01 \%$ of the image is used for the neighborhood.

- Transformation of the image into a black and white image using a threshold function after adjusting the image intensity.

For the purposes of the project, 112 samples of metal plates were provided by the contracting authority. At least one of the previously described approaches worked sufficiently with the majority of the provided samples. More detailed results are summarized at the end of the paper. Some examples are shown in Fig. 7.

As a next step of the processing, the positioning of the abscissae is automatically performed using a black and white image gained by one of the previously mentioned approaches. The correct positions of the abscissae are necessary for the application of equation (1). Column coordinates of the abscissae are known since the number of them is defined by the user (should be equal or bigger than 6 according to ISO 4628-8) and their positions should be distributed uniformly. The row coordinates are found simply as the transitions from black to white, starting from the top and bottom of the image.

The last step of the processing consists of possible manual manipulation with the abscissae to correct their possitions. This manipulation is sometimes necessary to get reliable results and it definitely rises the robustness of the device. A human

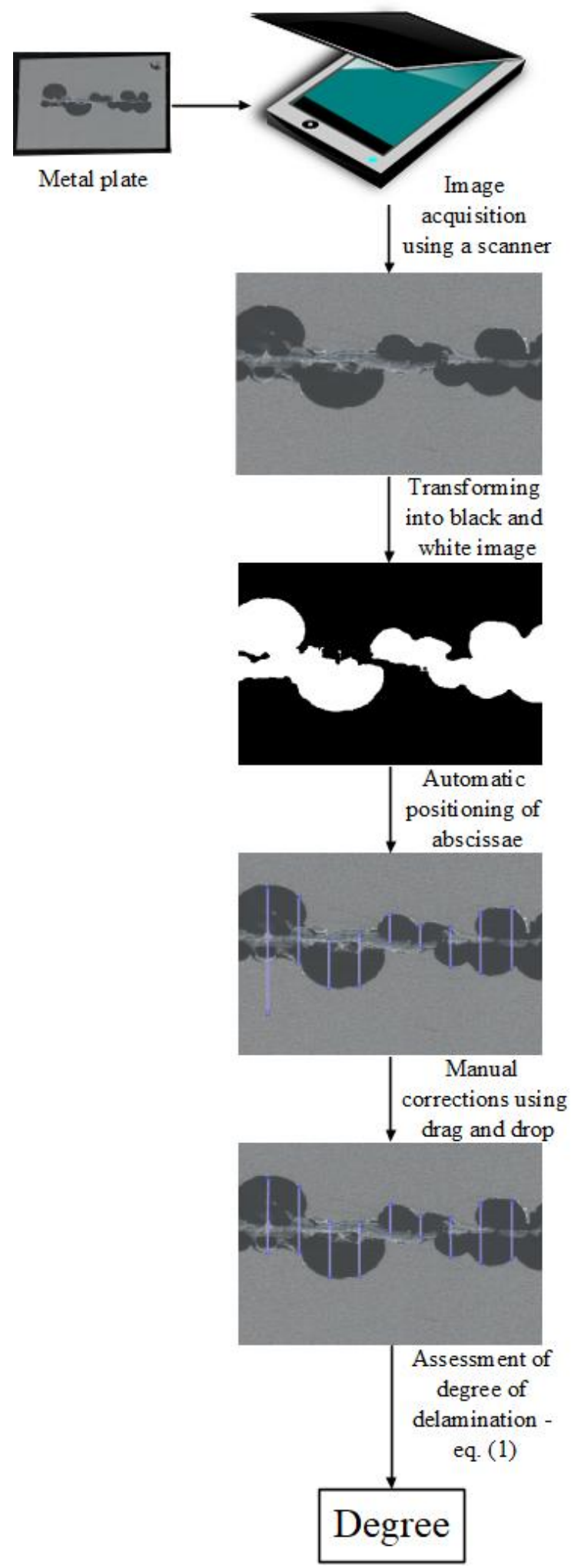

Fig. 6. Block diagram of intended device. 

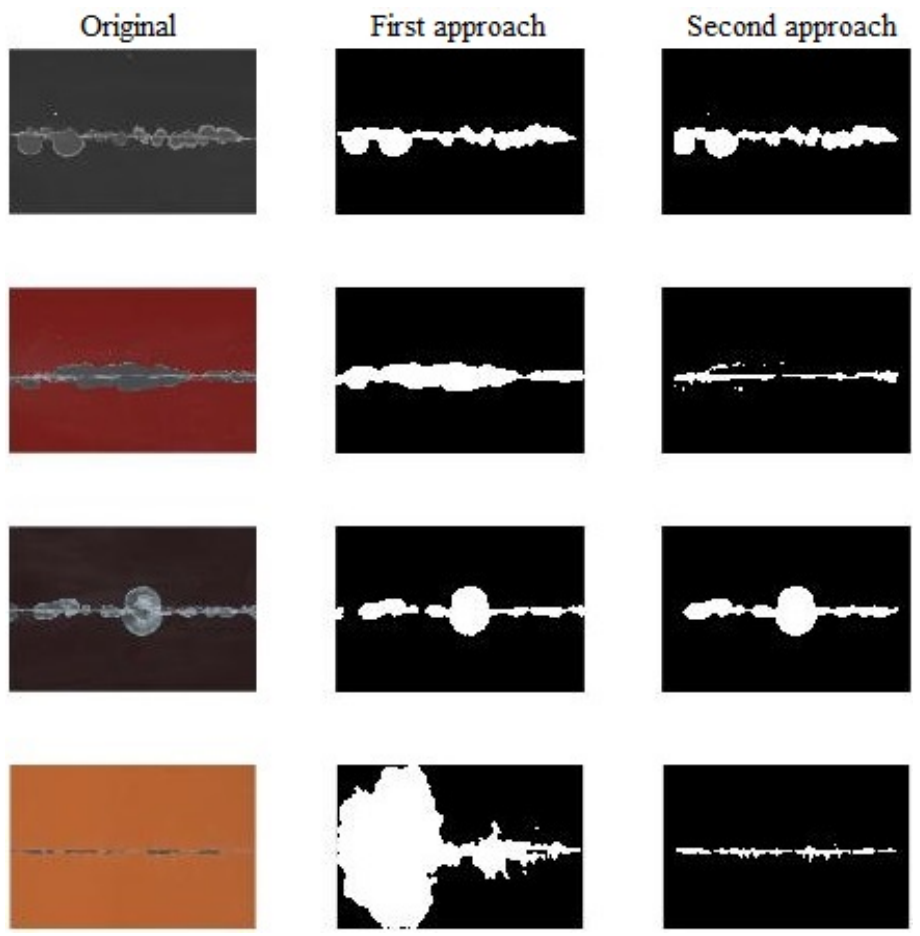

Fig. 7. Transformation into black and white image. Most of the samples were transformed sufficiently (see first and third row). In several cases, only one approach provided good results (see second and fourth row).

operator can manipulate the abscissae using a drag and drop approach with a computer mouse. The main human-computer interface of the device is shown in Fig. 8.

\section{CASE STUdY}

A prototype of a device proposed in previous sections was constructed and tested with an available dataset. A color scanner, which is used for image acquisition, provides $24 \mathrm{bit}$ RGB 3600x2400px images. Image processing is performed by a standard personal computer. The results obtained with 112 metal plates provided by the contracting authority are summarized in the table below. Three types of results are defined there:

- $\quad$ Sufficient evaluation with both approaches means that the sample is automatically evaluated correctly regardless of the approach selected for transformation into a black and white image

- $\quad$ Sufficient evaluation with at least one approach means that the sample is automatically evaluated correctly with at least one approach selected for transformation into a black and white image (includes previous subset)

- Human operator intervention required means that neither of approaches provided a correct solution and it was necessary to correct the positions of abscissae manually.

Note, that the dataset contains various kinds of coatings including different colors, surfaces of diverse granularity, or paintings with scattered reflectance. On the other hand, the number of samples is not entirely predicative. Thus, consider these results as an initial insight into the issue rather than a comprehensive statistical analysis. From the contracting authority perspective, the device is being applied in an operating laboratory with very positive feedback.

\section{TABLE I. SUMMARY OF THE RESULTS}

\begin{tabular}{|l|r|}
\hline Sufficient evaluation with both approaches & $68 \%$ \\
\hline Sufficient evaluation with at least one approach & $91 \%$ \\
\hline Human operator intervention required & $9 \%$ \\
\hline
\end{tabular}

\section{CONCLUSION}

The device, which implements the autonomous assessment of the degree of delamination around a scribe according to ISO $4628-8$, is proposed and tested in this contribution. The device was designed on demand and its first functional prototype is already used by the contracting authority.

The proposed procedure implements the optical device for data acquisition, two ways of image processing and provides a possibility of manual correction of the results. The preliminary evaluations of the results indicate, that more than $90 \%$ of the samples are interpreted using full automation, while the rest of the samples required operator intervention. Apparently, the prototype is fully open to further improvements, which will be definitely implemented with an extension of the dataset.

\section{ACKNOWLEDGMENT}

The work has been supported by the Funds of University of Pardubice, Czech Republic. This support is very gratefully acknowledged. We would also like to show our gratitude to METAL TRADE COMAX, one of the first representatives in continuous coil coating in Europe. METAL TRADE COMAX made the initial request to create the device for the autonomous assessment of the degree of delamination around a scribe and also provided the dataset of metal plates.

\section{REFERENCES}

[1] "Paints and varnishes - Evaluation of degradation of coatings - Designation of quantity and size of defects, and of intensity of uniform changes in appearance - Part 1: General introduction and designation system," International Organization for Standardization, Geneva, CH, Standard, Jan. 2016.

[2] J. Hanus, "Selection and evaluation of singlelayer coating compositions in corrosive environments," Acta Universitatis Agriculturae et Silviculturae Mendelianae Brunensis, vol. 59, no. 5, pp. 53-64, 2011.

[3] M. Whitfield, D. Bono, L. Wei, and K. Van Vliet, "High-throughput corrosion quantification in varied microenvironments," Corrosion Science, vol. 88, pp. 481-486, 2014.

[4] K. Choi and S. Kim, "Morphological analysis and classification of types of surface corrosion damage by digital image processing," Corrosion Science, vol. 47, no. 1, pp. 1-15, 2005.

[5] P. Kapsalas, M. Zervakis, and P. Maravelaki-Kalaitzaki, "Evaluation of image segmentation approaches for non-destructive detection and quantification of corrosion damage on stonework," Corrosion Science, vol. 49, no. 12, pp. 4415-4442, 2007.

[6] E. C. Cringasu, A. Dragomirescu, and C. A. Safta, "Image processing approach for estimating the degree of surface degradation by corrosion," in 2017 International Conference on ENERGY and ENVIRONMENT (CIEM), Oct 2017, pp. 275-278.

[7] M. Dobrovolny, P. Bezousek, and M. Hajek, "Application of a cumulative method for car borders specification in image," Radioengineering, vol. 17 , no. 4, pp. 2.75-2.79, 2008 


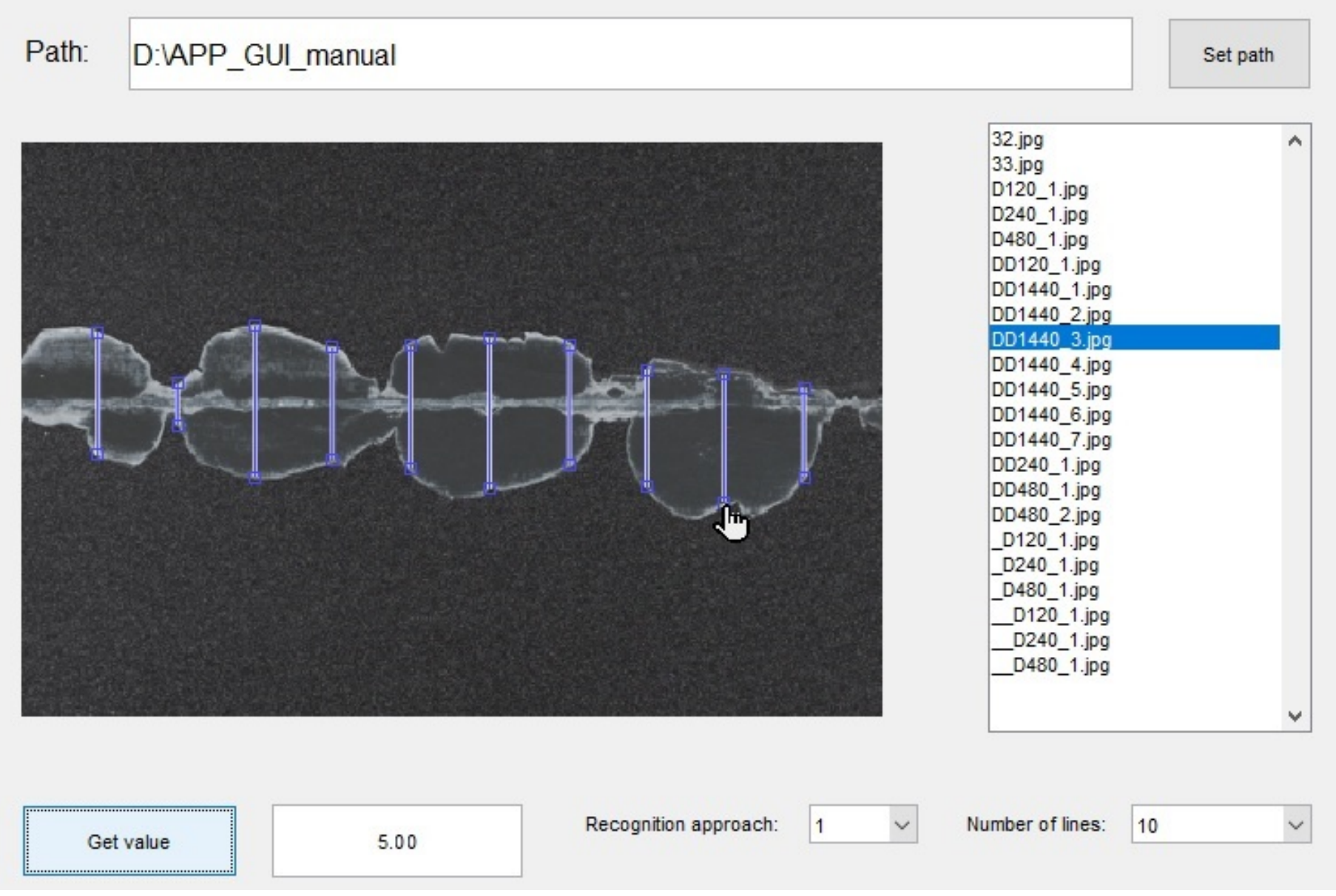

Fig. 8. Detail of human-computer interface.

[8] P. Skrabanek and F. Majerik, "Detection of grapes in natural environment using hog features in low resolution images," Journal of Physics: Conference Series, vol. 870, no. 1, 2017.

[9] S. Rahul, D. Honc, F. Dusek, and K. Gireesh, "Frontier based multi robot area exploration using prioritized routing," in 30th European Conference on Modelling and Simulation, ECMS 2016, June 2016, pp. 25-30.

[10] "Paints and varnishes - Evaluation of degradation of coatings - Designation of quantity and size of defects, and of intensity of uniform changes in appearance - Part 8: Assessment of degree of delamination and corrosion around a scribe or other artificial defect," International Organization for Standardization, Geneva, CH, Standard, Nov. 2012.

[11] T. F. Chan and L. A. Vese, "Active contours without edges," IEEE Transactions on Image Processing, vol. 10, no. 2, pp. 266-277, Feb 2001.

[12] J. S. Lim, Two-dimensional signal and image processing. Englewood Cliffs, N.J.: Prentice Hall, 1990. 